\title{
Repenser la formation professionnelle en muséologie : le cas du Québec
}

Rethinking museology training: the example of Québec

Yves Bergeron et Jennifer Carter

\section{OpenEdition}

1 Journals

Édition électronique

URL : http://journals.openedition.org/iss/557

DOI : $10.4000 /$ iss. 557

ISSN : 2306-4161

Éditeur

ICOM - International Council of Museums

Édition imprimée

Date de publication : 1 juin 2015

Pagination : 39-55

ISSN : 2309-1290

\section{Référence électronique}

Yves Bergeron et Jennifer Carter, «Repenser la formation professionnelle en muséologie : le cas du Québec », ICOFOM Study Series [En ligne], 43a | 2015, mis en ligne le 06 février 2018, consulté le 21 décembre 2020. URL : http://journals.openedition.org/iss/557 ; DOI : https://doi.org/10.4000/iss.557 


\title{
Repenser la formation professionnelle en muséologie : Le cas du Québec
}

\author{
Yves Bergeron \\ avec la collaboration de Jennifer Carter \\ Université du Québec à Montréal - Canada
}

\section{De la nécessité de repenser la formation}

II en va des programmes universitaires comme des expositions permanentes, ils sont rarement remis en question et renouvelés. Heureusement, les universités canadiennes ont mis en place au cours des dernières années des mécanismes qui les obligent à s'engager tous les dix ans dans un processus d'auto-évaluation. Cette politique s'impose d'autant plus que le monde des musées a changé en profondeur au cours des deux dernières décennies. Ceux qui suivent de près les grandes tendances savent bien que les musées se métamorphosent parce que le monde se transforme et que ces changements s'accélèrent entraînant des mutations profondes. Avant même d'entreprendre le processus d'autoévaluation du programme conjoint de la maîtrise en muséologie de I'Université du Québec à Montréal (UQAM) et de l'Université de Montréal (UdeM), nous savions pertinemment que le programme n'était plus en adéquation avec la réalité du monde muséal. Du même souffle, il nous est apparu clair que si nous ne repensions pas rapidement la formation en muséologie, compte tenu des transformations fondamentales qui bouleversent le monde muséal, les finissants qui sortent de nos universités ne seraient bientôt plus adaptés à la réalité de la pratique professionnelle. Cette crise devant les changements profonds du monde muséal convoque du même souffle des modifications majeures pour les universités.

S'il n'y a pas eu de véritables changements au sein des programmes au Québec, c'est en partie parce que les universités vivent en vase clos, qu'elles sont, il faut bien le reconnaître, trop souvent coupées de la réalité et qu'il est particulièrement complexe de changer des programmes existants en raison des longs processus d'évaluation, de consultation, d'analyse et de validation. II est plus simple de créer un programme que de modifier un programme existant. Cette évidence est d'autant plus flagrante quand on constate que I'Université du Québec en Outaouais a implanté en quelques mois un programme de maîtrise en muséologie ${ }^{3}$ qui innove et qui se révèle visiblement mieux adapté à la réalité professionnelle. En somme, le programme conjoint UQAM-UdeM se retrouve en quelque sorte dans une position qui nécessite des changements structurels. C'est pourquoi il nous semblait essentiel de mener une réflexion sur ce qui a changé dans l'univers des musées et sur les orientations que devrait prendre un nouveau programme de muséologie.

\footnotetext{
${ }^{3}$ Programme ouvert aux étudiants en septembre 2013. Maîtrise en muséologie et pratiques des arts offre quatre profils. "Muséologie " (Profil avec mémoire, 1614, Profil stage et essais, 1613), et "Pratiques des arts " (Profil projet de création et essai, 1611, Profil stages de production et essai, 1612).
} 


\section{La perspective nord-américaine}

Afin de mener une analyse juste et structurée de la pratique professionnelle, il convient de distinguer la réalité des trois grandes catégories de musées, c'est-à-dire les musées d'art, les musées de société (histoire, anthropologie, archéologie) et les musées de sciences (naturelles ou technologiques). À cet égard, le Québec s'est donné des outils qui permettent de catégoriser ces différentes institutions de manière à mener des enquêtes statistiques plus nuancées. L'Observatoire de la Culture et des Communications du Québec produit régulièrement des données statistiques sur la fréquentation des institutions muséales ${ }^{4}$ et les acquisitions par les musées. En parallèle de ces trois catégories d'institutions muséales, il nous semble essentiel de tenir compte des principales disciplines qui ont participé à la constitution du réseau des musées. II s'agit d'une clé majeure qui permet de saisir les enjeux liés à leur développement au cours des dernières décennies.

Contrairement à la tradition française où le statut de conservateur est soumis à une législation stricte et reconnu par des concours, la réalité est toute autre en Amérique du Nord où il n'existe pas de règles spécifiques pour obtenir le statut de conservateur. II n'existe donc pas de concours ou de cursus particuliers de sorte que les disciplines traditionnelles comme l'histoire de l'art, l'anthropologie, l'archéologie, l'histoire ou les sciences ont une plus grande influence sur la formation des conservateurs qui ont la responsabilité scientifique des collections. Comme les universités nord-américaines ont fait le choix d'une formation généraliste (Allard \& Lefebvre, 2001) ouverte aux trois catégories de musées, les programmes de muséologie forment plutôt des professionnels qui peuvent occuper différents postes dans les musées (conservateurs, éducateurs, guides, scénographes, chargés de projets, responsables des communications et du marketing, designers, webmestres...). Ces programmes ont par ailleurs permis la mise en place d'une culture commune, quelles que soient les diverses catégories de musées. Cette culture repose notamment sur l'importance de l'interprétation et une attention toute particulière pour les visiteurs. La préoccupation pour ceux-ci constitue sans aucun doute une des valeurs fondamentales qui traverse l'histoire depuis l'apparition des premiers musées en Amérique du Nord au début du XIX siècle.

Le statut de la muséologie comme discipline se révèle plus ambigu, car la tradition diffère d'un pays à l'autre. On croit souvent que le terme " muséologue » est apparu avec la création des programmes afin de désigner les chercheurs qui avaient le musée pour objet d'étude. Le terme s'est peu à peu imposé au Québec à partir de la décennie 1990 qui correspond avec l'arrivée des premiers professionnels diplômés de la maîtrise en muséologie. L'ambiguïté vient du fait que certains professionnels des musées revendiquent le statut de muséologue comme praticiens du champ muséal alors que les chercheurs qui œuvrent plutôt dans les universités revendiquent le statut de "muséologues" qu'ils associent à la recherche fondamentale. Bien que le Dictionnaire encyclopédique de la muséologie (Desvallées \& Mairesse, 2011) privilégie la perspective universitaire de la recherche, la réalité professionnelle se révèle plus

\footnotetext{
${ }^{4}$ Pour l'Observatoire de la Culture et des Communications du Québec, le concept d'«institutions muséales» regroupe les musées (art, histoire, ethnologie, archéologie, sciences naturelles et environnementales, sciences et technologie), les lieux d'interprétation et les centres d'exposition. Site consulté le 9 avril 2015.
} 
complexe. On pourrait très certainement formuler l'hypothèse que les universitaires qui ont mis en place les programmes de muséologie ont souhaité se démarquer du monde professionnel et de la fonction traditionnelle de conservateur pour se donner une nouvelle identité confirmant leur statut de chercheur. Bernard Schiele emprunte cette voie quand il tente de démontrer que la muséologie constitue fondamentalement un domaine de recherches (Schiele, 2012) qu'il oppose en quelque sorte à la pratique professionnelle. Pourtant, la réflexion sur la muséologie au Québec n'a pas pris naissance au sein des universités, mais plutôt dans le monde professionnel et plus particulièrement au sein de la Société des musées québécois (SMQ) et de l'Association des musées canadiens (AMC). Les premières publications définissant le champ de la muséologie et de l'interprétation du patrimoine ont émergé de Parcs Canada qui s'est doté au début de la décennie 1980 d'une politique de recherche et de publications permettant de construire une véritable réflexion sur la médiation muséale. II faut également tenir compte d'une série d'ouvrages publiés à compter de 1990 par le Musée de la civilisation du Québec (MCQ) en collaboration avec la Société des musées du Québec (Côté, 1991 ; Viel \& de Guise, 1992 ; Côté, 1992]. Le MCQ a joué à cet égard un rôle fondamental en devenant, grâce à son directeur général, Roland Arpin, le premier musée québécois à rayonner à l'international en raison de l'originalité de son concept muséal. Cette reconnaissance s'est structurée autour d'une série de textes et de publications de celui-ci (Bergeron, in press).

\section{À l'origine du programme de maîtrise à Montréal : 1987}

Après plusieurs années de consultations amorcées à la fin des années 1970 (Jentel, 1978), la Société des musées québécois et les instances du gouvernement du Québec constatent que les professionnels des musées ne disposent pas d'une formation adéquate en comparaison avec le Canada anglais et les autres pays. L'Université de Toronto est la seule université à offrir un programme de muséologie au Canada, inauguré en 1969 peu de temps après le centenaire de la Confédération canadienne (1967). Les célébrations entourant le dit centenaire ont été l'occasion pour le gouvernement fédéral de développer le réseau muséal canadien en créant notamment des musées régionaux et en élargissant le réseau de musées de sites de Parcs Canada (Lothian, 1987). II faut bien préciser que le monde des musées repose alors sur la contribution de bénévoles et d'amateurs du patrimoine (Bergeron Y., Dumas S., Thibault, M-T., 2007). Dans son rapport déposé à la Société des musées québécois en 1983, Jean Trudel reprenait une des conclusions de la politique québécoise de développement culturel rendue publique en 1978 :

\footnotetext{
Jusqu'à maintenant, les musées ont souvent été animés par des bénévoles dont le dévouement n'avait d'égal que l'amour de l'art et du patrimoine. On doit aujourd'hui y trouver aussi des professionnels sensibilisés non seulement aux problèmes de conservation, de restauration et d'éducation, mais encore aux activités d'interprétation, de recherche, d'administration. Dans cette perspective, le ministère des Affaires culturelles a entrepris une étude sur les besoins de formation muséologique au Québec. Les résultats de cette étude permettront de préciser des programmes de perfectionnement pour le personnel déjà en poste dans les musées et de mettre en marche, en accord avec le ministère de l'Éducation et les
} 
établissements d'enseignement, des programmes de formation afin d'assurer une relève apte à diversifier le réseau muséologique. Ainsi sera rendue possible la création de centres d'interprétation et d'établissements d'un genre nouveau du type de l'éco-musée. (La politique québécoise, 1978, pp. 362-363)

Jean Trudel ajoute à cette orientation du ministère des Affaires culturelles que le rapport Jentel soulignait dès 1978 le manque de formation dans les musées d'État : "le personnel est insuffisant et n'a pas toujours reçu la formation muséologique nécessaire, puisqu'une telle formation complète ne se donnait pas au Québec» (Jentel, 1978, p. 57). Marie-Odile Jentel souligne également « une connaissance très faible de la langue anglaise " qui coupe le personnel des musées de la littérature du réseau des musées nordaméricains. Cette littérature est notamment constituée de manuels et d'ouvrages sur la gestion des musées.

Au cours de la décennie 1980, la formation en muséologie pose toujours problème au pays. En 1984, le gouvernement canadien commande à Lee Jolliffe un rapport sur la formation en cours de carrière des muséologues au Canada (Jolliffe, 1984). Le rapport formule 26 propositions et souligne notamment que « la formation ne peut suffire à fournir assez de muséologues qualifiés pour combler les postes de cadres dans les musées. Cependant, un système national de formation en cours de carrière pourrait améliorer la situation-» (Joliffe, 1984, p. ii). Ce scénario privilégie donc la formation des professionnels au sein des musées qui pourrait être complétée par des programmes ponctuels et spécifiques offerts par les associations nationales et provinciales.

\section{La bataille des universités}

Au milieu des années 1980, il semblait évident que l'Université Laval obtiendrait le programme de maîtrise en muséologie. Le programme d'ethnologie et d'ethnographie de l'Université Laval (Bergeron, 2002) formait déjà des spécialistes de la culture matérielle et plusieurs de ces diplômés occupaient des postes de conservateurs dans les musées et le réseau de Parcs Canada ${ }^{5}$. Depuis la création des Archives de folklore en 1944, l'Université Laval se démarquait par des programmes de recherche en patrimoine et en histoire de l'art. Au moment de la création du programme, on retrouvait à Québec le Musée national des beaux-arts du Québec, le bureau régional de Parcs Canada avec son laboratoire de restauration, le Centre de Conservation du Québec et le Musée de la civilisation qui préparait son ouverture. Afin de contrer la position de I'Université Laval, I'UQAM et I'UdeM choisirent de s'allier afin d'offrir un programme conjoint. Ainsi, le Ministère des Affaires culturelles du Québec et les responsables des musées québécois ${ }^{6}$ proposaient en 1986 la création d'un programme conjoint de muséologie entre l'Université du Québec à Montréal et l'Université de Montréal (UQAM \& UdeM, 1986). Si cette alliance s'est révélée stratégique, elle n'en était pas pour autant organique. Chaque université conserva sa propre culture et le programme conjoint proposa une direction distincte pour chaque université.

Lorsque le programme de maîtrise en muséologie a ouvert ses

\footnotetext{
${ }^{5}$ Parcs Canada a créé en 1911 un réseau de lieux historiques nationaux et de parcs nationaux sur l'ensemble du territoire. On retrouve à Parcs Canada des équipes de chercheurs (historiens, archéologues, anthropologues, muséologues, interprètes) qui gèrent un réseau de musées de sites (167) et des collections nationales majeures.

Protocole signé entre l'UQAM et l'UdeM le 9 octobre 1986.
} 
portes aux premiers étudiants en 1987, chaque université pouvait compter sur des professeurs qui se concentraient autour de trois disciplines complémentaires dans chaque université. À l'UQAM, on retrouvait un noyau de professeurs en histoire de l'art', en communication $^{8}$ et en éducation ${ }^{9}$. À l'UdeM, les professeurs étaient rattachés aux départements d'histoire de l'art, d'anthropologie et de sciences. Il est indéniable que l'histoire de l'art a notamment contribué à structurer le programme puisque ce sont des historiens de l'art qui ont majoritairement enseigné et dirigé le programme. II faut d'ailleurs souligner la contribution à la muséologie des historiens de l'art de I'UQAM dont Raymond Montpetit ${ }^{10}$, Fernande SaintMartin $^{11}$, Louise Letocha ${ }^{12}$, Pierre Mayrand ${ }^{13}$ et Laurier Lacroix ${ }^{14}$. À cette liste, il faut ajouter d'autres professeurs d'histoire de l'art à I'UQAM qui avaient travaillé dans des musées ou qui réalisaient régulièrement des expositions. Mentionnons simplement Robert Derome et Michel Lessard ${ }^{15}$. À l'UdeM, Jean Trudel qui avait occupé le poste de conservateur dans de grands musées d'art au Québec ${ }^{16}$ a également joué un rôle de premier plan en devenant le premier directeur du programme.

Force est de constater que la perspective de l'histoire de l'art a été déterminante et qu'elle a favorisé une certaine continuité avec la tradition européenne. Cependant, il faut souligner que le programme devait nécessairement tenir compte de la composition du réseau des musées québécois. Quand on examine la répartition des trois grandes catégories d'institutions muséales, on constate que les musées d'histoire, d'ethnologie et d'archéologie représentent $45 \%$ de l'ensemble, que les institutions muséales en sciences comptent pour $38 \%$ et que les musées d'art constituent $17 \%$ du réseau. II est important de noter que la répartition de la fréquentation annuelle (Routhier, 2014) représentait 14,2 millions de visiteurs lors de l'enquête de l'Observatoire de la Culture et des Communications du Québec réalisée en 2013. En distribuant la fréquentation selon ces trois grandes catégories, les institutions muséales en histoire représentent $45 \%$ de la fréquentation annuelle. Ils sont suivis par les musées de sciences à $38,7 \%$ et les musées d'art à $18 \%$. De manière plus spécifique, la fréquentation scolaire suit cette même tendance. Elle se répartit comme suit : $45 \%$ dans les institutions vouées à I'histoire, $37 \%$ dans les institutions muséales scientifiques et $18 \%$ aux institutions consacrées aux arts. Compte tenu de ces résultats, il est par ailleurs étonnant de constater l'absence

\footnotetext{
${ }^{7}$ Raymond Montpetit, Laurier Lacroix, Louise Letocha et Pierre Mayrand.

${ }^{8}$ Bernard Schiele.

${ }^{9}$ Michel Allard, fondateur du Groupe de recherche en éducation muséale (GREM).

${ }^{10}$ Raymond Montpetit fut l'artisan du programme conjoint de muséologie UQAMUdeM, fondateur du programme (1986-1987) et directeur (1993 à 1999).

${ }_{11}$ Fernande Saint-Martin fut théoricienne de l'art et critique d'art avant de diriger le Musée d'art contemporain de Montréal (1972-1977).

${ }^{12}$ Louise Letocha dirigea le Musée d'art contemporain de Montréal de 1977 à 1982.

${ }^{13}$ Pierre Mayrand fut le premier muséologue québécois reconnu à l'international. II fut particulièrement actif au sein du Conseil international des musées (ICOM) et il participa à la création du Mouvement international pour la nouvelle muséologie (MINOM).

${ }_{4}^{4}$ Laurier Lacroix a participé à la création du programme de maîtrise en muséologie. Le gouvernement du Québec lui a décerné en 2008 le prix Gérard-Morisset en patrimoine.

${ }^{15}$ Michel Lessard a publié de nombreux ouvrages sur le patrimoine québécois. On lui doit notamment La nouvelle encyclopédie des antiquités du Québec aux Éditions de l'Homme en 2007.

${ }^{16}$ Formé à l'École du Louvre, Jean Trudel a été conservateur au Musée du Québec, premier directeur francophone du Musée des beaux-arts de Montréal en 1977 et président de la Société des musées québécois de 1979 à 1981.
} 
d'historiens ${ }^{17}$ au sein du programme tant à l'UQAM qu'à l'UdeM. Ce constat est d'autant plus étonnant que le Canada s'est distingué dès la décennie 1970 par le développement du réseau de Parcs Canada dont l'expertise en interprétation de l'histoire a été rapidement reconnue à l'international. II est intéressant de noter qu'on compte au Canada et aux États-Unis des programmes de deuxième cycle d'histoire appliquée (Public History) centrés sur la médiation.

Les statistiques de fréquentation reflètent bien la réalité des musées au Canada et aux États-Unis où les musées d'histoire et de société sont les plus nombreux et les plus fréquentés. Contrairement à l'opinion du grand public qui associe les musées aux arts, la réalité nord-américaine se révèle bien différente. II devenait donc impératif que la formation de deuxième cycle en muséologie tienne compte de la réalité du réseau muséal. C'est pourquoi le programme devait considérer la perspective de l'histoire, de l'ethnologie et de l'archéologie. Du même souffle, on ne pouvait négliger les musées de sciences naturelles et les musées de sciences et technologies qui se classent au second rang dans la fréquentation des institutions muséales.

\section{Une perspective fonctionnaliste et une culture commune de la muséologie}

Le recul du temps nous permet de constater que le programme conjoint de muséologie de I'UQAM et de I'UdeM s'est d'abord structuré dans une approche fonctionnaliste. II s'agit d'un choix qui répondait alors aux besoins de la communauté muséale de la décennie 1980. On retrouve les traces de cette approche dans les besoins exprimés en 1992 par la Société des musées québécois qui offrait des formations spécialisées (Boucher \& Lapointe, 1992) depuis 1979.

Au milieu des années 1980, il y a donc près de 25 ans, le ministère de l'Éducation fait le choix de favoriser la formation en muséologie au deuxième cycle. Ainsi, les étudiants se concentrent au premier cycle dans les disciplines associées à la muséologie (histoire de l'art, histoire, anthropologie, archéologie, sciences) et développent leur formation en muséologie au deuxième cycle. Ce choix fait en sorte que le programme accepte des étudiants provenant de divers horizons et que ceux-ci acquièrent une culture commune de la muséologie à travers huit séminaires. C'est pourquoi la formation s'est d'abord concentrée non pas sur des approches disciplinaires, mais sur les fonctions muséales: conservation (5), exposition (2), éducation (2) et gestion (1). Certains séminaires sont cependant centrés sur des catégories spécifiques de musées afin de permettre aux étudiants de se spécialiser. Mentionnons notamment les musées d'art (MSL6510 L'art contemporain et MSL6509 Muséologie et histoire de l'art), les musées d'histoire (MSL6510 Interprétation et lieux historiques, MSL6516 Le musée et les pratiques du discours historique), les musées de sciences (MSL6506 Muséologie des sciences et des techniques, MSL6515 Les collections de sciences

\footnotetext{
17 À l'exception de quelques historiens sensibles à la médiation de l'histoire dans les musées (Paul-André Linteau, Alain Beaulieu et Joanne Burgess), les départements d'histoire se sont peu investis dans les programmes de muséologie à Montréal. Le contexte fut diamétralement opposé à l'Université Laval où le DESS de muséologie s'est développé au sein du département des sciences historiques qui regroupe des historiens, des historiens de l'art, des ethnologues, des archéologues et des archivistes.
} 
naturelles et leur mise en valeur), les musées d'archéologie (MSL6521 Muséologie et archéologie) et les musées d'ethnologie (MSL6503 Ethnomuséologie).

Outre les catégories de musées, un séminaire thématique est consacré aux nouvelles muséologies (MSL6104), ainsi qu'aux musées régionaux et au tourisme (MSL6512), qui concernent majoritairement les musées d'histoire et de société. Afin de compenser les limites de l'offre de cours initiale, l'UQAM et l'UdeM se sont dotées d'un séminaire à contenu variable qui a permis d'expérimenter de nouvelles thématiques. C'est ainsi que s'est mis en place à l'UdeM le séminaire de l'École du Louvre ${ }^{18}$ qui permet aux étudiants de s'initier à la muséologie européenne. Dans la même perspective, le séminaire offert par l'UQAM sur la muséologie nordaméricaine ${ }^{19}$ offre une immersion dans des musées américains.

Dès la création du programme, se sont ajoutées de nouvelles disciplines qui traduisaient également les préoccupations de la muséologie au milieu des années 1980. L'éducation muséale (Allard 2012), et de manière plus large la médiation ${ }^{20}$, s'est imposée comme une des caractéristiques de la muséologie nord-américaine qui valorise l'interprétation à travers le travail des guides et animateurs auprès de différentes catégories de visiteurs. Du même souffle, les changements amorcés dans les musées avec la libéralisation de l'économie imposaient un développement marqué des stratégies de communication et de marketing ${ }^{21}$ afin de développer de nouveaux publics. Aux spécialistes de la communication se sont ajoutés les sociologues qui ont développé des outils de mesure de la fréquentation et de la satisfaction des visiteurs. Cette préoccupation pour les visiteurs s'est traduite par l'arrivée de nouveaux professionnels dans les musées qui avaient pour objet d'étude l'expérience de visite (Montpetit \& Bergeron, 2009) des différentes catégories de publics.

\section{Montréal - Québec}

On oublie trop souvent que la décision stratégique de l'UQAM et de l'UdeM de se regrouper a eu pour effet la création d'un second programme de deuxième cycle en muséologie à l'Université Laval alors que le gouvernement du Québec avait fait le choix d'opter pour un seul programme que l'on souhaitait d'ailleurs contingenté dans chacune des deux universités à 20 étudiants par année. Déçu de la position du ministère de l'Éducation, le recteur de l'Université Laval, Michel Gervais, décide de créer en 1988 un DESS en muséologie. Pour mettre en place ce nouveau programme, il choisit comme professeur Philippe Dubé qui venait tout juste de participer à l'ouverture du Musée de la civilisation en réalisant l'exposition permanente Mémoires (Bergeron \& Dubé, 2009). Philippe Dubé s'était précédemment distingué par son travail de conservateur au musée régional de Charlevoix et à Parcs Canada. Le DESS s'est

\footnotetext{
18 Ce séminaire est particulièrement populaire et est offert à chaque session d'automne.

${ }_{19}$ La première expérience a été menée en 2012 à la Smithsonian Institution à Washington, DC.

${ }^{20}$ Raymond Montpetit a précisé le concept de médiation et plus particulièrement le concept d'interprétation dans la muséologie nord-américaine. Voir Montpetit, 2011. II fait notamment référence à l'ouvrage de Freeman Tilden (1957).

${ }_{21}$ Le directeur du Musée de civilisation, Roland Arpin (1987-2001), a été l'un des premiers directeurs a affirmer dès la fin des années 1980 la nécessité pour les musées de tenir compte des publics par la mise en place de services de communication et de marketing. Voir à ce sujet deux chapitres dans Roland Arpin, (1997) : "Culture et marketing : une liaison dangereuse ?» p. 167-195; "Le musée entre son public et ses publics », p. 225-242.
} 
retrouvé associé au département des sciences historiques de l'Université Laval qui regroupe les programmes suivants: histoire, histoire de l'art, ethnologie, muséologie, archéologie et archivistique. Compte tenu de la structure du département, on comprend que le programme de muséologie s'est plutôt orienté vers les musées d'histoire et de société. En ce sens, le DESS de l'Université Laval offrait aux futurs muséologues un profil différent de la maîtrise à I'UQAM et à l'UdeM. Dès lors, l'Université Laval opte pour une scolarité centrée sur quatre séminaires la première année alors que la seconde année est consacrée au stage et à la rédaction d'un court mémoire de sorte que la durée effective des études à Québec comme à Montréal est de deux ans.

II est important de souligner que les deux programmes sont restés marqués depuis 1988 par la compétition traditionnelle entre les villes de Montréal et de Québec de sorte qu'il y a eu peu d'échanges au cours des 25 dernières années à l'exception de quelques projets de recherche menés conjointement par Raymond Montpetit (UQAM) et Philippe Dubé. La reconnaissance des deux programmes s'est confirmée lors de la création en 2001 du Prix Roland-Arpin qui souligne l'excellence des meilleurs étudiants en muséologie (Dionne, 2010).

Si le programme de maîtrise forme annuellement près de 40 étudiants (20 à l'UQAM et 20 à l'UdeM), le DESS de l'Université Laval accueille en moyenne une dizaine d'étudiants par année. Si on considère qu'une large part des étudiants de la maîtrise provient de l'étranger, ce sont environ 25 étudiants québécois qui obtiennent le diplôme en moyenne chaque année, ce qui représente peu de nouveaux professionnels quand on sait que le réseau compte plus de 3800 années-personnes ${ }^{22}$ pour une masse salariale de 166,5 M\$ (Bergeron \& Dumas, 2007).

\section{Oublis et zones d'ombre}

Ce qui est frappant avec le recul des années, c'est que certaines perspectives étaient absentes des programmes de formation en 1987. Aucun séminaire n'abordait la problématique du patrimoine ${ }^{23}$, alors que les conventions internationales et les politiques du patrimoine ont élargi les responsabilités des musées à l'égard du patrimoine matériel et notamment du patrimoine immatériel à compter de 2003. Ce choix s'explique certainement par le désintérêt pour le concept de patrimoine après le référendum de 1980 sur la souveraineté du Québec. La notion de patrimoine a été réhabilitée en 2000 avec le rapport Arpin qui proposait un nouveau projet de loi sur le patrimoine à la ministre de la Culture, Agnès Maltais.

De même, l'intérêt grandissant pour les publics et les pratiques

\footnotetext{
${ }^{22}$ Le terme " année-personne " provient du terme anglais person-year utilisé par I'Institut de la statistique afin de mesurer précisément le temps de travail dans le réseau muséal. "Unité de mesure correspondant au travail que peut accomplir une personne pendant un an, deux personnes pendant six mois et ainsi de suite, et qui sert, dans le budget, à répartir les crédits affectés à la main-d'œuvre.» Office québécois de la langue française (OQLF) https://www.oqlf.gouv.qc.ca/ressources/bibliotheque

/dictionnaires/terminologie_relations_professionnelles/anneepersonne.htmnsulté le 10 avril 2015.

${ }^{23} \mathrm{Ce}$ constat s'explique probablement par un désintérêt généralisé pour le patrimoine après le référendum de 1980 sur la souveraineté du Québec. Le terme même de patrimoine disparaît dès lors des programmes universitaires.
} 
culturelles qui a contribué à transformer en profondeur les relations que les musées entretiennent avec les publics ne figurait pas dans la liste des séminaires offerts en muséologie. II s'agit pourtant d'un des faits saillants de la muséologie québécoise qui s'est confirmé avec l'ouverture du Musée de la civilisation en 1988 qui s'était donné un service de la recherche dont l'un des axes était notamment consacré aux publics et à la satisfaction des visiteurs. II serait aujourd'hui impensable d'offrir une formation en muséologie qui n'aborderait pas la problématique des publics et des pratiques culturelles. C'est pourquoi il devient nécessaire d'intégrer l'expertise développée en sociologie de la culture.

Cette préoccupation pour les publics a conduit les musées nordaméricains à privilégier le développement de services de communication et de marketing à compter de la décennie 1990. La contribution de chercheurs en sciences de l'information devenait donc incontournable. Le musée est devenu un véritable média de masse et ne peut plus être considéré du strict point de vue des collections.

Les technologies figurent au cœur des mutations ayant contribué à transformer les musées. L'informatisation des collections dans un premier temps, puis leur numérisation, ont contribué à favoriser un accès démocratique aux objets patrimoniaux. Le développement de plates-formes numériques a permis le développement d'expositions virtuelles et de musées virtuels. Les diverses expériences réalisées dans cette perspective au cours des deux dernières décennies ont permis d'ouvrir la diffusion des musées sur de nouvelles platesformes. Si les technologies n'ont pas suscité l'attention de ceux qui ont créé les programmes de muséologie au milieu des années 1980, comment envisager maintenant la formation en négligeant les dimensions technologiques?

Si un seul séminaire est consacré à la gestion des musées, cela semble bien peu compte tenu des transformations observées dans la gouvernance de ceux-ci. La nécessité de développer de nouvelles sources de revenus et la création de fondations exigent un approfondissement des principes de gouvernance des musées. Les relations avec les conseils d'administration, les communautés et les divers paliers de gouvernements doivent reposer sur une véritable réflexion sur la gestion des institutions muséales. Contrairement à ce que l'on croit trop souvent, les musées ne relèvent pas uniquement de la culture, ils sont indissociables de l'économie et du politique.

Certaines grandes tendances internationales influencent également le développement des musées. Ainsi, la convergence des musées, des archives et des bibliothèques (LAMS) a également contribué à modifier en profondeur la gestion des collections et du patrimoine favorisant ainsi une approche globale de la culture. Cette tendance montre bien que c'est le rapport à la culture qui s'est transformé au cours des dernières décennies. Ainsi, comment pouvons-nous envisager la recherche et plus spécifiquement la documentation des collections et le partage de l'information dans cette nouvelle perspective sans intégrer l'archivistique et la bibliothéconomie à la formation en muséologie?

La multiplication des législations qui concernent aujourd'hui la gestion des objets muséaux et patrimoniaux fait en sorte que les professionnels des musées ne peuvent plus ignorer les lois nationales, les législations internationales, les grandes conventions 
sur la culture et le patrimoine pas plus que les politiques culturelles développées par les régions et les municipalités. Mentionnons plus précisément la loi sur le droit d'auteur, le droit à l'image et les codes de déontologie. Dans la pratique, les professionnels doivent tenir compte de ces cadres de référence et si les grands musées disposent des services d'avocats, ce n'est pas le cas pour les petits et moyens musées qui constituent l'essentiel du réseau muséal. La question qui se pose aujourd'hui est de savoir comment intégrer au cursus une connaissance adéquate des législations et conventions qui encadrent la gestion des musées et du patrimoine.

\section{La muséologie aujourd'hui}

Élise Dubuc a proposé en 2012 une réflexion sur les fonctions muséales (Dubuc, 2012) qui permet d'envisager plus largement les fonctions traditionnelles des musées: conservatoire, culturelle, sociale, économique, scientifique, politique, éducative et symbolique. Cette nouvelle nomenclature pourrait éventuellement permettre de restructurer la formation en muséologie.

Tenant compte des récentes transformations dans l'univers des musées, toutes catégories confondues, de nouvelles disciplines se sont ajoutées au fil des années. Ainsi, il est impossible maintenant de faire abstraction du tourisme, du droit, de l'archivistique et de la bibliothéconomie. Les musées sont devenus des leviers économiques pour le développement régional qui se structure autour du tourisme (« Nouveaux musées... », 2008). L'internationalisation des relations des musées et la circulation des objets de collections obligent les musées à une plus grande vigilance à l'égard des lois (Bergeron, Letocha, Grimard, 2006) sur le droit d'auteur, le droit à l'image et la propriété intellectuelle. De même, on ne peut plus négliger les lois sur l'exportation et la circulation des biens culturels de même que les conventions internationales sur le patrimoine culturel et le patrimoine culturel immatériel. Ces impératifs législatifs ne peuvent plus être négligés dans la formation en muséologie.

Par ailleurs, l'informatisation des collections s'est complexifiée et tend à se normaliser en fonction de règles internationales qui intègrent à la fois les principes de l'archivistique et de la bibliothéconomie. À ce chapitre, la muséologie contrairement à ces deux disciplines avait fait des choix différents. Pendant très longtemps, chaque musée développait ses propres normes et ses propres outils de catégorisation des objets de sorte que l'informatisation des collections au début de la décennie 1990 a posé des problèmes majeurs de normalisation (Bergeron, 1996) afin que les données puissent être compatibles d'un musée à l'autre. Au Canada, le réseau canadien d'information sur le patrimoine (RCIP) a joué un rôle clé en définissant des règles communes. Dans le même esprit, le réseau Info-Muse créé au sein de la Société des musées québécois a doté les musées d'outils méthodologiques pour l'informatisation des collections. II faut donc envisager la formation à la lumière des outils internationaux de normalisation et de diffusion de l'information.

Le développement des technologies a ouvert de nouvelles perspectives pour la diffusion hors musée dans le cyberespace. Le "musée virtuel » a donné naissance à des formes nouvelles de médiation. L'Université du Québec en Outaouais a d'ailleurs initié en 2007 un certificat en cybermuséologie. Aucun musée ne peut 
négliger cette forme de communication s'il veut attirer d'autres publics.

Enfin, on ne peut passer sous silence la prise en compte par le Conseil international des musées du patrimoine immatériel dans la nouvelle définition du musée. La convention internationale pour la sauvegarde du patrimoine culturel immatériel (PCI) adoptée par I'UNESCO en 2003 a contribué à transformer en profondeur la mission des musées. Les musées canadiens et plus particulièrement les musées québécois sont particulièrement concernés par cette orientation puisque la nouvelle loi sur le patrimoine culture adoptée par le gouvernement du Québec en 2012 confie de nouveaux mandats aux institutions muséales. Cette législation qui remplace la loi sur les biens culturels intègre de nouvelles responsabilités. La loi inclut maintenant «les paysages culturels patrimoniaux, le patrimoine immatériel, ainsi que les personnages, les évènements et les lieux historiques ". Les municipalités se voient confier de nouvelles responsabilités « dans la protection et la mise en valeur du patrimoine, en leur donnant davantage de pouvoirs ». Enfin, le gouvernement a choisi d'offrir "des pouvoirs aux communautés autochtones, notamment pour l'attribution de statuts légaux aux éléments du patrimoine culturel qui leur sont chers » (Loi patrimoine, 2014). C'est dans cet esprit que les musées québécois doivent maintenant composer avec cette loi. En somme, les musées ont la responsabilité de développer leurs politiques de développement des collections en tenant compte à la fois du patrimoine matériel et du patrimoine culturel immatériel. Ce passage majeur a été souligné par la Société des musées du Québec en 2010 avec la tenue du colloque sur le patrimoine culturel immatériel (Musées et patrimoine immatériel, 2010).

L'intérêt grandissant pour le patrimoine s'est traduit dans la grande majorité des pays industrialisés par l'adoption de nouvelles politiques visant la protection et la mise en valeur de nouvelles formes de patrimoines (culture matérielle et objets contemporains, patrimoine immatériel, paysages, langues, coutumes, traditions). Dans la pratique professionnelle, le clivage entre muséologie et patrimoine tend à disparaître de sorte que ces deux univers convergent l'un vers l'autre.

Le statut de commissaire qui s'est imposé au cours des deux dernières décennies a également contribué à transformer la pratique professionnelle dans les musées. En faisant appel à des commissaires (Esse, 2011) indépendants pour réaliser des expositions on a modifié le statut traditionnel du conservateur (musées et collections, 2010) qui a été remis en question par l'arrivée des commissaires et des chargés de projets responsables des expositions. On assiste donc depuis deux décennies à un clivage entre les professionnels responsables des collections (conservateurs) et les nouveaux conservateurs (commissaires et chargés de projets) qui se consacrent aux expositions et à la médiation.

Le mouvement de mondialisation qui touche l'univers de la culture se traduit par la multiplication des expositions internationales produites par des consortiums de musées qui imposent de nouvelles alliances stratégiques. Cette tendance s'inscrit dans la perspective du développement d'une économie de marché. II ne fait aucun doute que la mondialisation a contribué à creuser un écart considérable entre les petits musées proches de leurs communautés et les 
musées nationaux qui disposent de moyens importants leur permettant de créer des alliances économiques avec d'autres grands musées et de présenter des expositions de type «blockbuster ". Dans cette perspective, la formation doit prendre en considération ces deux réalités distinctes.

Enfin, les nouvelles technologies et plus particulièrement la culture numérique ont donné naissance à une dématérialisation de l'objet patrimonial et muséal induisant de nouvelles règles de conservation, de documentation et de diffusion des collections.

On observe également que les frontières traditionnelles entre les catégories de musées tendent à se fondre, voire à disparaître. Les expositions recourent à des muséographies apparentées dans les musées d'art, les musées de société et les musées de sciences. On constate une forme de métissage (Bergeron, 2010) dans les musées qui autrefois occupaient des champs spécifiques et qui optaient pour des muséographies distinctes.

\section{Repenser la formation : nouveaux défis}

Compte tenu de ces tendances et des changements qu'elles induisent dans la pratique muséale, on comprend bien qu'il devient nécessaire de repenser la formation en muséologie. La question qui se pose aujourd'hui aux responsables des programmes de muséologie est de savoir comment on peut concilier cette nouvelle nébuleuse que représente le champ muséal où convergent diverses disciplines. Bien que nous n'en soyons pas encore à l'étape de proposer un nouveau modèle de formation, il ne fait aucun doute qu'il faut convoquer de nouvelles disciplines afin de couvrir le vaste terrain qui balise aujourd'hui le champ muséal. Comme nous l'avons démontré précédemment, certaines disciplines doivent aujourd'hui être prises en considération. Nous ne saurions former les futurs muséologues sans qu'ils ne soient initiés adéquatement aux approches de l'ethnologie à l'égard de la culture, de la bibliothéconomie et de l'archivistique pour la gestion des collections ou du droit pour la bonne gestion du patrimoine.

II paraît tout aussi essentiel de distinguer la formation en fonction de deux perspectives, c'est-à-dire la pratique professionnelle dans les musées et la recherche fondamentale de la muséologie. Ces deux perspectives ne peuvent cependant être envisagées comme étant isolées ou antagonistes. Les universités tentent depuis trois décennies de circonscrire et de s'approprier symboliquement le champ muséal en voulant contrôler le statut de "muséologie » bien qu'il ait été créé par les professionnels qui avaient fait de la muséologie dans la seconde moitié du $20^{\mathrm{e}}$ siècle un champ disciplinaire à part entière. Rappelons simplement que la muséologie québécoise n'a pas été reconnue dans un premier temps par la spécificité de la formation universitaire, mais par l'excellence et l'innovation d'institutions muséales canadiennes et québécoises ${ }^{24}$.

\footnotetext{
${ }^{24}$ La muséologie québécoise a d'abord été reconnue sur la scène internationale par l'excellence de la muséographie et des techniques d'interprétation de Parcs Canada au milieu de la décennie 1970. L'ouverture du Musée de la civilisation demeure pour le Québec le moteur de la reconnaissance de la muséologie québécoise à compter de 1988. Le MCQ est le premier musée québécois à exporter à l'international son concept muséologique et sa manière toute particulière de structurer le musée. Voir à ce sujet : Arpin, 1992.
} 


\section{Savoirs, savoir-faire et savoir-être}

Jusqu'ici, la formation en muséologie s'est concentrée sur l'acquisition de savoirs techniques et théoriques liés à la pratique professionnelle dans les musées. Ces savoirs comme nous l'avons démontré se structurent autour des principales fonctions du musée (préservation, médiation et gestion). La mise en place d'un cursus qui se termine par un stage et la rédaction d'un travail dirigé permet l'expérimentation des savoir-faire. Chaque étudiant a l'occasion de s'initier à la culture des musées. Cependant, rien dans le cursus ne prépare les futurs professionnels à la troisième dimension des savoirs qui est probablement la plus importante, c'est-à-dire les " savoir-être professionnels ". II est essentiel de reconnaître que les musées ne constituent pas simplement des institutions administratives au service des gouvernements ou des citoyens selon leur mission. En réalité, ce sont des institutions publiques dont la mission correspond à des valeurs partagées par un groupe spécifique. En d'autres termes, chaque musée possède sa propre culture et ses propres valeurs. L'acquisition des "savoir-être » au moment de la formation universitaire permet de mieux connaître et de pouvoir ainsi intégrer pleinement la culture spécifique de chaque institution muséale. L'acquisition du " savoir-être " peut notamment s'inscrire par une meilleure intégration des stages et par un programme de mentorat. La Société des musées québécois a notamment expérimenté en 2006 et en 2011 un programme de mentorat comme outil de développement professionnel (SMQ, 2012). La SMQ reconnaît que le mentorat répond à six besoins spécifiques : soutien aux jeunes professionnels, développement de compétences, soutien aux gestionnaires, maintien en emploi, transitions professionnelles, planification et développement de carrière. On rappelle que le mentorat permet de favoriser le sentiment d'appartenance à un musée, de réduire la mobilité du personnel, de préparer la relève et d'améliorer la communication et le travail en équipe.

Au sein du programme de maîtrise, nous avons mené au cours de la dernière année un projet pilote de mentorat pour les étudiants nouvellement inscrits en les jumelant à des professeurs de muséologie dont le profil de carrière correspondait à leurs aspirations professionnelles. II reste maintenant à évaluer les effets de ce programme sur les étudiants. Nous croyons que le mentorat représente un véritable défi pour la formation professionnelle dans le cadre universitaire qui s'est peu préoccupée jusqu'ici de l'intégration professionnelle. En d'autres termes, le défi des universités ne consiste pas essentiellement à former de jeunes professionnels maîtrisant des connaissances, mais à les préparer à s'intégrer pleinement à la culture des institutions muséales.

\section{Une avenue à envisager : le " musée-école »}

Afin de pouvoir trouver un équilibre entre la théorie et la pratique, la perspective de structurer la formation en muséologie autour du concept de "musée-école » semble une avenue à considérer. À cet égard, il existe plusieurs corrélations à faire avec d'autres formations universitaires. Pensons simplement à la formation des comédiens, des scénographes, des designers, des archéologues ou des médecins. Comment envisager la formation des comédiens sans que ceux-ci ne puissent acquérir les bases de leur pratique professionnelle en dehors des théâtres? C'est pourquoi les programmes de théâtre offrent l'infrastructure nécessaire à la formation et à la pratique professionnelle. II en va de même pour les scénographes. Dans la même perspective, les universités qui offrent 
des formations en archéologie ont mis en place le modèle du " chantier-école ${ }^{25}$ où les jeunes archéologues peuvent intégrer à la fois la théorie et la pratique. Le même principe s'applique dans la formation en médecine où les étudiants partagent leur temps entre la formation théorique et des séjours prolongés dans des hôpitaux universitaires. Cette pratique de l'externat qui consiste à l'acquisition d'une formation auprès d'un médecin-professeur dans un hôpital universitaire repose en même temps sur le principe du mentorat. L'Université de Montréal, par exemple, structure l'externat autour de 12 stages obligatoires et de stages à option. Au cours de ces stages, les étudiants en médecine consacrent une partie de leur temps à des gardes en milieu hospitalier aidant à développer les compétences cliniques, en particulier la prise de décision, la reconnaissance de l'urgence d'une situation et le sens des responsabilités. Cette formation est combinée à l'apprentissage au raisonnement clinique (ARC). Les médecins expérimentés transmettent autre chose de plus essentiel que les savoirs et qui relève plutôt du " savoir-faire » et du "savoir-être ". Ne devrait-il pas en être de même pour la formation en muséologie?

\section{Recherche et création}

Nous croyons que les programmes de muséologie doivent également reconnaître que le travail de muséologie se partage entre deux axes qui se révèlent à la fois distincts et complémentaires. Si la muséologie s'enseigne avant tout en considérant la dimension de la recherche fondamentale, la réalité professionnelle nous révèle que le travail des muséologues implique une large part de création. C'est d'ailleurs ce qui fait de la muséologie une discipline hybride et unique. On ne saurait former les jeunes professionnels sans reconnaître cette réalité et donner des outils afin de concilier ces deux dimensions. Un nouveau programme devrait préparer les jeunes muséologues à comprendre que la réussite professionnelle dépend largement de l'intégration partagée entre recherche et création.

\section{Conclusion}

L'auto-évaluation de la maîtrise en muséologie représente une occasion de repenser la formation en muséologie, mais cet exercice permet également au monde universitaire de repenser son rapport avec le champ muséal. Pour les universitaires, la question n'est plus de déterminer si la muséologie constitue une discipline en soi. La question centrale repose davantage sur une réflexion en profondeur sur le monde des institutions muséales qui se révèle être un univers complexe et mouvant auquel les universités ont du mal à s'adapter. On ne peut plus proposer un seul profil aux étudiants alors que des secteurs de la pratique professionnelle exigent de plus en plus des formations approfondies en gestion des collections, en éducation muséale, en médiation numérique, en droit, en étude des publics et des pratiques culturelles et globalement en recherche.

L'autre défi auquel les universités doivent faire face consiste à trouver un équilibre fragile entre la vision universitaire du musée centré sur la recherche alors que les musées sont en même temps des lieux de création et de productions culturelles. Ne faut-il pas

\footnotetext{
${ }^{25}$ On peut penser au laboratoire d'archéologie et au chantier école de l'Université Laval. Voir : /www.laboarcheologie.ulaval.ca/ L'Université de Montréal a développé un chantier de l'École de fouilles archéologiques en partenariat avec le Musée de Pointeà-Callière.
} 
penser la formation en muséologie en terme de « recherche et de création »? II ne fait aucun doute que dans un monde en pleine mutation il faut tout mettre en œuvre pour permettre l'émergence d'une « nouvelle muséologie » à la mesure du « Nouveau Monde » dans lequel les musées évoluent.

\section{Références}

Altshuler, B., Leshchenko, A., Riccio, R., Sandell, R., Schwarzer, M., Small, N. P., Sorin, G. S., \& Stapp, C. (2011). "How Do Museum Studies Programs Address the Nature of Museums Today?" The Exhibitionist, 30 (1), Spring 2011, 82-86.

Allard, M., \& Lefebvre, B. (2001). La formation en muséologie et en éducation muséale à travers le monde. Québec: MultiMonde.

Allard, M. (2012). « Le parcours et les perspectives du champ et de la recherche en éducation muséale. " Dans La muséologie, champ de théories et de pratiques, (pp. 121-130). Montréal: Presses de I'Université du Québec.

Arpin, R. (1992). Le Musée de la civilisation. Concept et pratiques. Québec: MultiMonde.

Arpin, R. (1997). Des musées pour aujourd'hui. Québec: Musée de la civilisation.

Arpin, R. (président du groupe-conseil). (2000). Un présent du passé. Proposition de politique du patrimoine culturel déposé à Agnès Maltais, ministre de la Culture et des Communications du Québec. Québec, Groupe-conseil sur la politique du patrimoine culturel.

Boucher, L., \& Lapointe, L., (1992). "Évaluation des besoins en matière de perfectionnement », Musées, 14 (2), 7-13.

Bergeron, Y. (1996). " Les systèmes de classification et l'informatisation des collections: enjeux et déroutes. " Revue d'histoire de la culture matérielle, 43. Ottawa: Musée national des sciences et de la technologie, 19-33.

Bergeron, Y. (2002). "Le complexe des musées d'ethnographie et d'ethnologie au Québec 1967-2002 », Ethnologies, Musées / Museums, 24 (2), 47-77.

Bergeron, Y. (2010). "Métissage entre musées d'art et musées de société. » Culture \& Musées, 16: Actes Sud, 45-63.

Bergeron, Y., Dusseault-Letocha, L., \& Grimard, J. (2006). « Cadre juridique de l'univers du patrimoine, des institutions muséales et des archives. "État des lieux du patrimoine des institutions muséales et des archives. Cahier 1: Premier regard, Québec, Observatoire de la culture et des communications du Québec, (pp. 29-32.). Page consultée le 17 mai 2015, au http://www.stat.gouv.qc.ca/statistiques /culture/patrimoine-musees-archives/cahier-01-etatdeslieux.pdf

Bergeron, Y., Dumas, S., \& Thibault, M.-T. (2007). État des lieux du patrimoine des institutions muséales et des archives. Cahier 3 : Les instititutions muséales du Québec, redécouverte d'une réalité complexe, Québec, Observatoire de la culture et des communications du Québec. Page consultée le 17 mai 2015, au http://www.stat.gouv.qc.ca/statistiques/culture/patrimoine-museesarchives/cahier -03-etatdeslieux.pdf

Bergeron, Y., \& Dubé, P. (Dir.). (2009). Mémoire de Mémoires. L'exposition inaugurale du Musée de la civilisation. Québec: les Presses de l'Université Laval.

Bergeron, Y., Arsenault, D. \& Provencher-St-Pierre, L. (Eds.). (2015). Musées et muséologies: Au-delà des frontières Les muséologies nouvelles en question. Québec: les Presses de l'Université Laval.

Bergeron, Y. (Dir.). (à paraître). Un nouveau musée pour un Nouveau monde. La muséologie selon Roland Arpin. Paris: L'Harmattan

Côté, M. (Dir.). (1991). Musées et gestion. Québec: Musée de la civilisation, Université Laval.

Côté, M. (Dir.). (1992). Tendances de la muséologie au Québec. Québec: Musée de la civilisation, Société des musées québécois, Environnement Canada, Service des parcs.

Desvallées, A., \& Mairesse, F. (Dir.). (2011). Dictionnaire encyclopédique de 
muséologie. Paris: Armand Colin.

Dictionnaire de données des sciences humaines du RCIP. Page consultée le 15 avril 2014, au www.pro.rcip-chin.gc.ca/bd-dl/ddrcip_shchindd hs-fra.jsp

Dionne, H. (2010). "Le Prix Roland-Arpin ou l'avenir en héritage. » Roland Arpin. Visions culturelles. Québec: Musée de la civilisation, pp. 1519.

Dubuc, É. (2012). « La professionnalisation des musées et l'autonomisation des programmes d'enseignement. "Dans La muséologie, champ de théories et de pratiques. (pp. 153-164). Montréal: Presses de l'Université du Québec.

Entente entre l'Université du Québec à Montréal et l'Université de Montréal, 9 octobre 1986, $6 \mathrm{p}$.

Esse (2011). "Be Nice to your Curator / Commissaires Curators." Esse, 72, printemps/été.

Guide pratique Le mentorat comme outil de développement professionnel, (2012). Montréal: Société des musées québécois. Page consultée le 15 avril 2014, au www.musees.qc.ca/pdf/smq_guide_pratique_mentorat.pdf

Hedegaard, R. (2004). "The benefits of archives, libraries and museums working together: a Danish case of shared databases." New Library World, 105 (7/8), 290-296.

Jentel, M.-O. (1978). Rapport sur la formation muséologique au Québec, Direction des musées privés et centres d'exposition. Québec: Ministère des Affaires culturelles, Québec.

Jolliffe, L. (1984). La formation en cours de carrière des musélogues au Canada, rapport préparé pour le Programme d'appui aux musées des Musées nationaux du Canada. Ottawa: Musées nationaux du Canada.

Le livre blanc des musées de France, (2010). Musées et collections publiques de France, 127.

La loi sur le patrimoine culturel en bref. Page consultée le 14 avril 2014 à partir de www.mcc.gouv.qc.ca/index.php?id=5121.

Lothian, W. F. (1987). Petite histoire des parcs nationaux du Canada. Ottawa: Environnement Canada Parcs.

Montpetit, R., \& Bergeron, Y., avec la collaboration d'Anik Meunier et d'Amélie Giguère (2009). L'expérience des visiteurs dans les sites historiques. Rapport final. Québec: Parcs Canada.

Musées et patrimoine immatériel (2010). Musées (Québec), 29 (numéro spécial).

Le livre blanc des musées de France (2010). Musées et collections publiques de France, 260, $127 \mathrm{p}$.

"Nouveaux musées, nouveaux tourismes », (2008). Téoros, 27(3) (numéro spécial).

La politique québécoise de développement culturel, 2 : Les trois dimensions d'une politique: genres de vie, création, éducation. (1978). (pp. 362-363). Québec: Editeur officiel du Québec.

Regourd, M. (Dir.). (2013). Musées en mutation. Un espace public à revisiter. Paris: L'Harmattan.

Routhier, C. (2014). « La fréquentation des institutions muséales en 2012 et 2013. " Optique Culture, 17, Page consultée le 15 avril 2014, au www.stat.gouv.qc.ca/statistiques/culture/bulletins/optique-culture17.pdf

Schiele, B. (2012). "La muséologie un domaine de recherches. " In A. Meunier (Dir.). La muséologie champ de théories et de pratiques (pp. 79-100). Montréal: Presses de l'Université du Québec.

Simon, N. (2010). The participatory museum. Santa Cruz, CA: Museum 2.0

Tilden, F. (1957). Interpreting Our Heritage. Chapel Hill: University of North Carolina Press.

Viel, A., \& de Guise, C. (Dir.). (1992). Muséo-séduction, Muséo-réflexion. Québec: Musée de la civilisation, Environnement Canada, Service des parcs.

\section{Résumé}

Vingt-cinq ans après la création du premier programme de 
formation en muséologie au Québec (1988), l'Université du Québec à Montréal et l'Université de Montréal s'engagent dans une réflexion sur la refonte de leur programme conjoint de maîtrise. Après deux décennies de mutations profondes dans l'univers des musées (Hedegaard, 2004 ; Simon, 2010 ; Altshuler et al., 2011; Regourd, 2013 ; Bergeron et al., 2015), comment repenser la formation des jeunes professionnels afin qu'ils puissent composer avec ces transformations qui concernent à la fois de nouveaux objets de collections et de nouvelles technologies qui transforment la nature même des collections et qui conséquemment posent des défis de conservation et de médiation. L'analyse propose une approche conceptuelle des transformations de l'univers des musées, un bilan de la formation et ses effets sur la pratique professionnelle au Québec et au Canada.

Mots clé : formation professionnelle, muséologie, Québec

\section{Abstract}

Rethinking museology training: the example of Québec

Twenty-five years after the first graduate program in museology was founded in Québec in 1988, I'Université du Québec à Montréal and the Université de Montréal are engaging in a deep reflection on the reform of their joint Master's program. Following two decades of profound changes within the museum world (Hedegaard, 2004; Simon, 2010; Altshuler, 2011; Regourd, 2013; Bergeron et al, 2015), how should we be thinking about the training of young professionals in light of transformations that have led to both new types of objects entering museum collections and new technologies that are themselves transforming the very nature of these collections and thus are challenging traditional conservation methods and interpretation practices?

Key words: professional training, museology, Québec 